\title{
Failure of Percutaneous Endoscopic Resection of a Renal Cystic Nephroma on Longer-Term Follow-Up
}

\author{
J. Stuart Wolf, Jr., M.D. ${ }^{1}$ and Atreya Dash, M.D. ${ }^{2}$
}

\begin{abstract}
In 2005 we reported in this journal the treatment of cystic nephroma, a rare but presumed benign renal tumor, with percutaneous resection. Initial follow-up confirmed removal of the intra-pelvic portion of the cystic nephroma and persistence of the unresected intra-parenchymal portion. Surveillance with computed tomography revealed gradual regrowth of the mass, ultimately resulting in a size greater than that at the time of initial resection. Laparoscopic radical nephrectomy without adrenalectomy revealed benign cystic nephroma.
\end{abstract}

\section{Introduction}

$I^{2}$

N 2005 WE REPORTED in this journal the treatment of cystic nephroma, a rare but presumed benign renal tumor, with percutaneous resection. ${ }^{1}$ A 53 -year-old healthy woman was evaluated for an incidental right renal mass extending into the collecting system (Fig. 1) with flexible ureteroscopy, biopsy, and aspiration. The findings suggested a benign renal cystic lesion. The patient requested the least invasive approach possible to firmly establish the diagnosis. We performed percutaneous endoscopic resection of the intra-pelvic portion of the mass with a $24 \mathrm{~F}$ resectoscope through a single upper pole access tract. Pathology confirmed cystic nephroma.

\section{Case Report}

The initial follow-up computed tomography (CT) images (Fig. 2) revealed removal of the intra-pelvic portion of the cystic nephroma and persistence of the unresected intra-parenchymal portion, such that the overall size of the mass was $3.5 \times 4.7 \mathrm{~cm}$, compared to $3.5 \times 6.2 \mathrm{~cm}$ prior to resection. Subsequent surveillance $\mathrm{CT}$ imaging, performed due to uncertainty about the natural history of cystic nephroma, revealed gradual regrowth of the mass, to $4.3 \times$ $5.1 \mathrm{~cm} 14$ months after resection, and $5.2 \times 6.6 \mathrm{~cm} 22$ months after resection (Fig. 3). After review of the case at our Multidisciplinary Urologic Oncology Conference, radical rather than partial nephrectomy was recommended. Laparoscopic radical nephrectomy without adrenalectomy was performed uneventfully and the patient was discharged on postoperative day 2. Serum creatinine 3 weeks postoperatively was $1.0 \mathrm{mg} / \mathrm{dL}$, compared to 0.7 $\mathrm{mg} / \mathrm{dL}$ at the time of initial resection and at the time of radical nephrectomy. Pathology again revealed cystic nephroma.

\section{Discussion}

Cystic nephroma is a rare cystic renal neoplasm characterized by multilocular cysts lined with epithelium but separated by fibrous septa. As noted in our prior report, partial nephrectomy has been successfully applied in this setting. To our knowledge, our case represented the first treatment of cystic nephroma using a percutaneous endoscopic approach, albeit without complete resection, since the intent of the procedure was definitive diagnosis rather than cure. Acknowledging the uncertainty about the natural history of cystic nephroma and the possibility that the residual mass might regrow, we followed the mass with CT imaging. Ultimately, because our percutaneous resection did not completely remove the lesion, the mass grew and surgical extirpation was still required. The scarring from prior percutaneous resection was a factor in de-

\footnotetext{
${ }^{1}$ University of Michigan, Department of Urology, Ann Arbor, Michigan.

${ }^{2}$ University of California Irvine Medical Center, Department of Urology, Orange, California.
} 


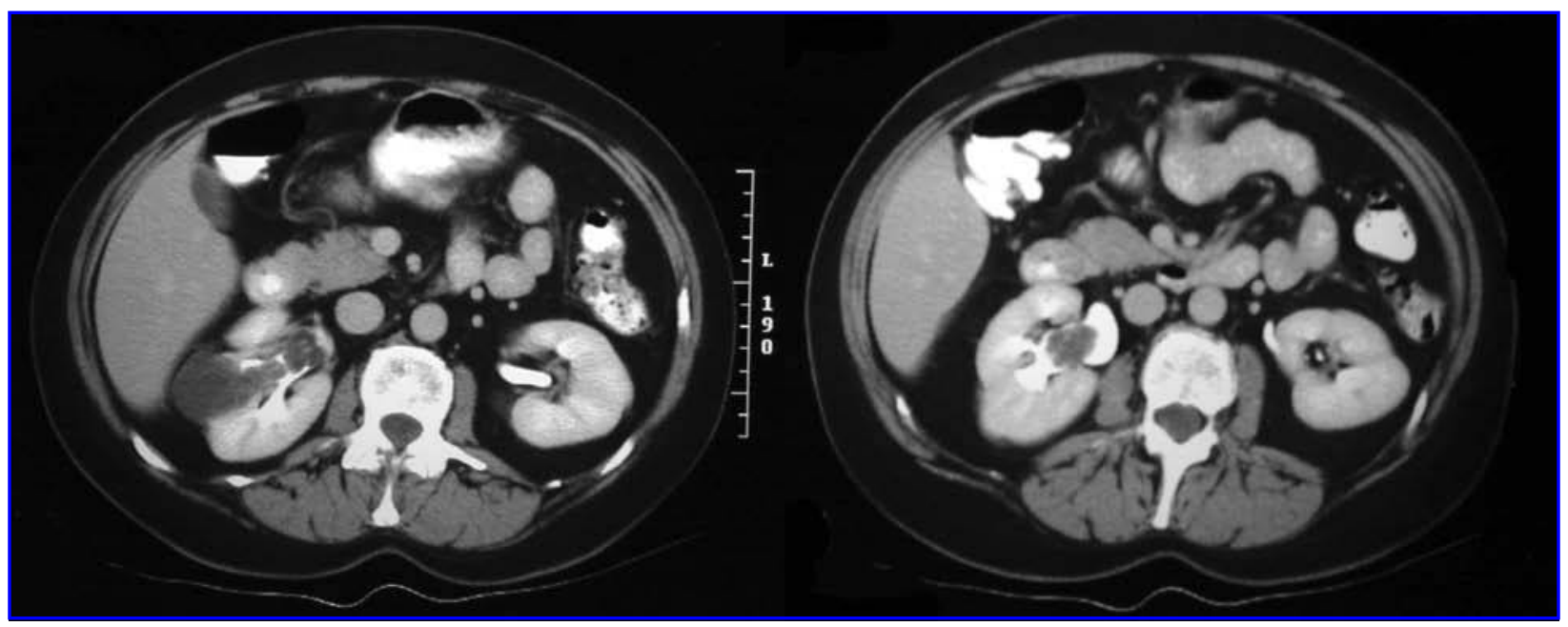

FIG. 1. Two computed tomography images reveal the cystic nephroma with extension into the renal pelvis. From Dash and Wolf. 1

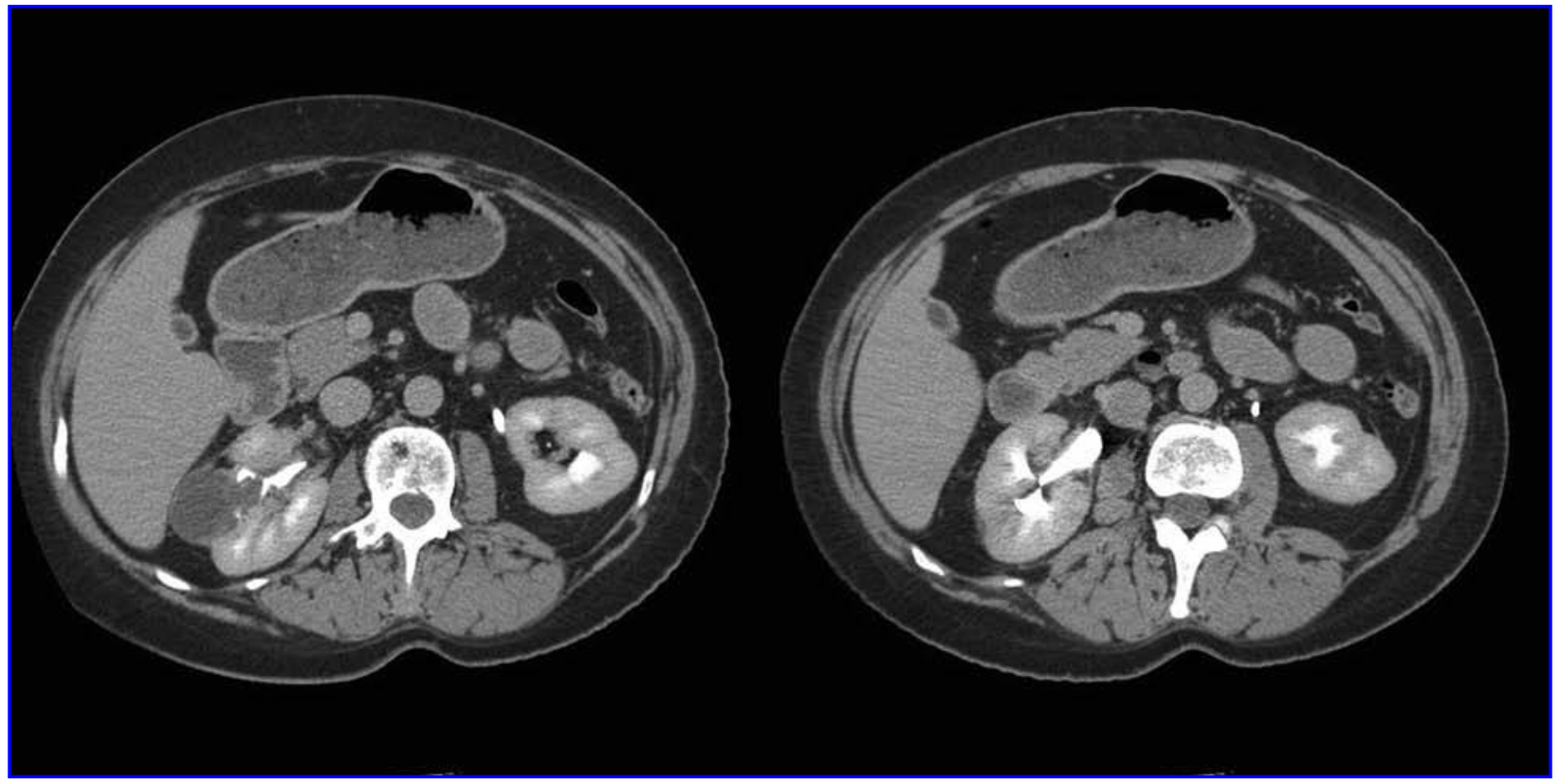

FIG. 2. The postprocedure computed tomography images (4 weeks postoperatively) confirm resection of the intra-pelvic portion of the cystic nephroma and persistence of its unresected intra-parenchymal portion. From Dash and Wolf. ${ }^{1}$

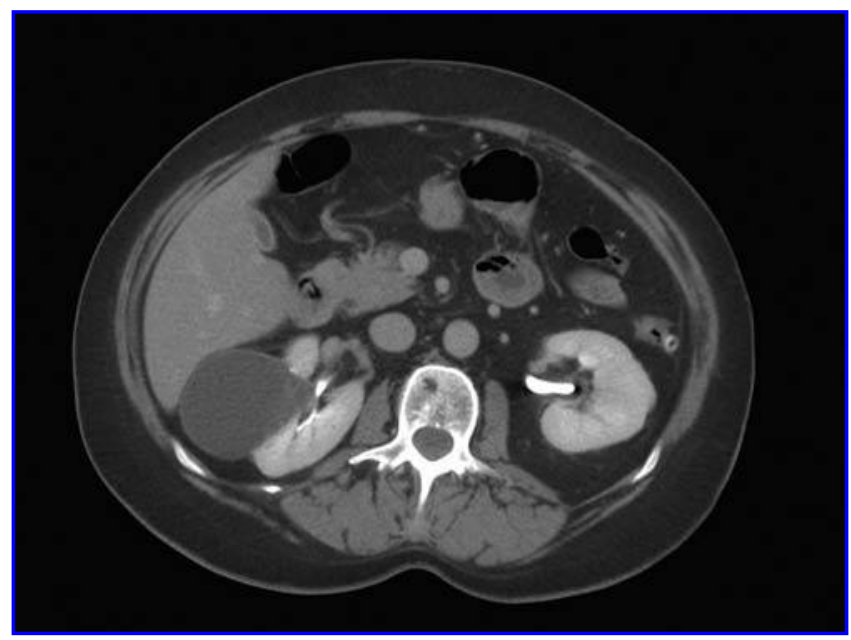

FIG. 3. Computed tomography image 22 months after percutaneous resection reveals enlargement of the intraparenchymal portion of the cystic nephroma. 
ciding on radical rather than partial nephrectomy, and although it still would have been a challenging procedure (Fig. 1), we might have elected to perform partial rather than radical nephrectomy if the percutaneous procedure had not been performed. This outcome needs to be considered when contemplating percutaneous resection of a cystic nephroma, unless all abnormal tissue can be removed.

\section{Reference}

1. Dash A, Wolf JS Jr. Percutaneous treatment of renal cystic nephroma. I Endourol 2005;19:724-725.
Address reprint requests to: J. Stuart Wolf, Jr., M.D. Department of Urology University of Michigan

TC 3875

1500 East Medical Center Drive Ann Arbor, Michigan 48109-0330

E-mail: wolfs@umich.edu

\section{Abbreviation Used}

$\mathrm{CT}=$ computed tomography 
